Online Appendix for

\title{
Reputation and Persistence of Adverse Selection in Secondary Loan Markets
}

\author{
V. V. Chari, Ali Shourideh, and Ariel Zetlin-Jones*
}

June 4, 2014

\section{Contents}

1 Normalization of Bank and Buyer Payoffs 2

2 General Properties of Static Equilibrium 2

3 Proof of Proposition $1 \quad 3$

4 Basic Properties of Partial Pooling Allocations 6

4.1 Establishing the constraint $(\mathrm{A} 13) \ldots \ldots \ldots \ldots$

5 Infinite Horizon Model with Divisible Assets 12

*Chari: Department of Economics, University of Minnesota, 4-101 Hanson Hall, 1925 Fourth Street South, Minneapolis, MN, 55455, and Federal Reserve Bank of Minneapolis (e-mail: chari002@umn.edu), Shourideh: Finance Department, The Wharton School, University of Pennsylvania, 2322 Steinberg-Dietrich Hall, 3620 Locust Walk, Philadelphia, PA (e-mail: shouride@wharton.upenn.edu), Zetlin-Jones: Tepper School of Business, Carnegie Mellon University, 5000 Forbes Avenue, Pittsburgh, PA 15213 (e-mail: azj@cmu.edu). 


\section{Normalization of Bank and Buyer Payoffs}

Suppose buyers offer contracts of the form $(x, \hat{t})$. If a bank accepts such a contract, the bank's payoffs are given by

$$
\hat{t}+(1-x)(\pi \bar{v}+(1-\pi) \underline{v}-c) .
$$

Buyers' profits from $(x, \hat{t})$ if a bank of type $\pi$ accepts are given by

$$
x(\pi \bar{v}+(1-\pi) \underline{v})-\hat{t} .
$$

Letting $t=\hat{t}-x \underline{v}$ and $v=\bar{v}-\underline{v}$, these payoffs can be expressed as

$$
t+(1-x)(\pi v-c)+\underline{v}
$$

and

$$
x \pi v-t .
$$

It is then immediate that we may normalize $\underline{v}$ and analyze the effect of changes in the collateral value, $\underline{v}$, by analyzing changes in the loan spread, $v$.

Q.E.D.

\section{General Properties of Static Equilibrium}

Here we show that any symmetric monotone equilibrium of the static model has the properties described in the text.

1. Suppose by way of contradiction that $x_{l}<1$. Consider a deviation to the contract $\left(x_{h}, t_{h}, x_{l}+\varepsilon, t_{l}+(\underline{\pi} v-c) \varepsilon\right)$. This deviation keeps the low-quality bank indifferent, whereas the profit per low type is higher and hence profitable.

2. Suppose that the following incentive constraint holds with strict inequality:

$$
t_{l}+\left(1-x_{l}\right)(\underline{\pi} v-c) \geq t_{h}+\left(1-x_{h}\right)(\underline{\pi} v-c)
$$

Then, combining the two incentive constraints, we must have $x_{h}<1$. A deviation to a contract $z^{\prime}=\left(x_{h}+\varepsilon, t_{h}^{\prime}+\varepsilon(\bar{\pi} v-c), x_{l}, t_{l}\right)$ leaves the utility of the high-quality bank unchanged and increases the profits per high-quality bank.

3. To prove that buyers make zero profits on each point in the support of $z$, first note that in any monotone equilibrium, for each pair of contracts offered by buyers $j$ and $-j$, $\delta_{j}\left(z_{j}, z_{-j} ; \bar{\pi}\right)=\delta_{j}\left(z_{j}, z_{-j} ; \underline{\pi}\right)$. Thus, in any monotone equilibrium, profits associated with a contract $z$ for buyer $j$ can be written as

$$
\Pi(z)=\left[\mu\left(x_{h} \bar{\pi} v-t_{h}\right)+(1-\mu)\left(x_{l} \underline{\pi} v-t_{l}\right)\right] \int \delta_{j}\left(z, z_{-j} ; \bar{\pi}\right) d F\left(z_{-j}\right) .
$$

Since the contract $(0,0,0,0)$ guarantees zero profits, it follows that for each $z \in \operatorname{Supp}(F)$ with $\int \delta_{j}\left(z, z_{-j} ; \bar{\pi}\right) d F\left(z_{-j}\right)>0$,

$$
\mu\left(x_{h} \bar{\pi} v-t_{h}\right)+(1-\mu)\left(x_{l} \underline{\pi} v-t_{l}\right) \geq 0 .
$$

Furthermore, in any mixed strategy, at each such $z$, profits must be identical, or $\Pi(z)=\bar{\Pi}$. We now prove that for each $z \in \operatorname{Supp}(F)$, (1) holds with equality. To do so, consider the 
lowest payoff of the low-quality bank,

$$
\underline{u}=\inf _{z \in \operatorname{Supp}(F)} t_{h}+\left(1-x_{h}\right)(\bar{\pi} v-c) .
$$

Let $\underline{z}$ denote the contract that attains this infimum. Suppose first that the support of $F$ has no mass at $\underline{z}$. Clearly, there is a sequence $\left\{z_{n}=\left(x_{h, n}, t_{h, n}, x_{l, n}, t_{l, n}\right)\right\}_{n=1}^{\infty} \subset S u p p(F)$ that converges to $\underline{z}$. Since banks accept the contract they prefer most, the probability that a contract in this sequence is accepted converges to zero, so that $\int \delta_{j}\left(z_{n}, z_{-j}\right) d F\left(z_{-j}\right) \rightarrow 0$. Hence, $\Pi\left(z_{n}\right) \rightarrow 0$ (since $\mu\left(x_{h, n} \bar{\pi} v-t_{h, n}\right)+(1-\mu)\left(x_{l, n} \underline{\pi} v-t_{l, n}\right)$ is bounded). Now, since $\Pi(z)=\pi, \forall z \in \operatorname{Supp}(F)$, we must have $\pi=0$.

Suppose next that $F$ has positive mass $f_{0}$ at $\underline{z}$. Given our tie-breaking rule, the contract $\underline{z}$ attracts the bank with probability of at most $f_{0} / 2$. Now, suppose by way of contradiction that at this contract, profits are strictly positive. Consider a deviation from $\underline{z}$ to the contract $z^{\prime}=$ $\left(x_{h}, t_{h}+\varepsilon, x_{l}, t_{l}+\varepsilon\right)$ for some small $\varepsilon>0$, where $\underline{z}=\left(x_{h}, t_{h}, x_{l}, t_{l}\right)$. The profits conditional on this offer being better than the contract offered by the other buyer are arbitrarily close to conditional profits under $\underline{z}$. Since this contract is strictly preferred to $\underline{z}$ by both types, the probability that the deviating contract is accepted is at least $f_{0}$. Since the probability that $\underline{z}$ is accepted is at most $f_{0} / 2$, it follows that expected profits strictly increase with this deviation. We have established a contradiction, so profits are zero.

4. Suppose that $t_{l}<\underline{\pi} v$ for some contract offered in equilibrium. A deviation to a contract $\hat{z}=(1, \bar{\pi} v-\varepsilon, 1, \underline{\pi} v-\varepsilon)$ for $\varepsilon$ sufficiently small makes positive profits from lowquality banks by attracting them with positive probability and possibly the high-quality banks as well.

Q.E.D.

\section{Proof of Proposition 1}

Consider the case where $\mu<\tilde{\mu}$. We begin by showing that in any pure strategy equilibrium, $t_{l}=\underline{\pi} v$. This result together with the four key properties implies that any pure strategy outcome must coincide with the least-cost separating outcome. Suppose, by way of contradiction, that a pure strategy equilibrium has $t_{l}>\underline{\pi} v$. Clearly if $x_{h}=0$, this contract makes negative profits, so $x_{h}>0$. Consider a deviating contract $\hat{z}=\left(x_{h}-\varepsilon, t_{h}-\varepsilon(\bar{\pi} v-c), 1, t_{l}\right)$ with $\varepsilon$ small. This deviating contract keeps the high-quality bank indifferent to the supposed equilibrium contract and relaxes the low-quality bank's incentive constraint. It attracts the high-quality bank with probability $1 / 2$ under our tie-breaking rule. Conditional on attracting the high-quality bank, profits from this bank rise by $\varepsilon c$ and profits from the low-quality bank are unchanged. Thus, profits are strictly positive, so we have a contradiction.

To show that the least-cost separating outcome is a pure strategy equilibrium, we establish a preliminary result regarding the high-quality bank's payoffs. Consider the set of offers $\left(x_{h}, t_{h}\right)$, to the high-quality bank implied by a binding incentive constraint for the low-quality bank and zero profits as a function of $t_{l}$. Since both the profit function and the incentive constraint are linear functions of $x_{h}, t_{h}$, and $t_{l}$, it follows that $x_{h}$ and $t_{h}$ are linear functions of $t_{l}$. Since the payoff function to the high-quality bank is linear in $x_{h}$ and $t_{h}$, this payoff, $\hat{u}_{h}\left(t_{l}\right)$, is also linear in $t_{l}$. Clearly, $\hat{u}_{h}(\underline{\pi} v)$ is equal to the high-quality bank's payoff in the least-cost separating outcome and $\hat{u}_{h}(\hat{p}(\mu))=\hat{p}(\mu)$. Since $\mu<\tilde{\mu}, \hat{u}_{h}(\underline{\pi} v)>\hat{u}_{h}(\hat{p}(\mu))$. Since $\hat{u}_{h}\left(t_{h}\right)$ 
is a linear function of $t_{h}$, it follows that $\hat{u}_{h}(\underline{\pi} v)>\hat{u}_{h}\left(t_{l}\right)$, so that the high-quality bank prefers the least-cost separating outcome to any associated with $t_{l}>\underline{\pi} v$ that breaks even and in which the incentive constraint for the low-quality bank holds with equality. Given this preliminary result, suppose that buyer 2 offers the least-cost separating outcome. Consider a deviation by buyer 1 to contracts of the form $\hat{z}=\left(\hat{x}_{h}, \hat{t}_{h}, 1, \hat{t}_{l}\right)$ with $\hat{t}>\underline{\pi} v$. Since any such contract that breaks even does not attract the high-quality bank, it is clear that any contract that does attract the high-quality bank must yield negative profits. Clearly since any deviation that has $\hat{x}_{l}<1$ is dominated by a further deviation that sets the amount sold by the low-quality bank to 1 and keeps it indifferent to the original deviation, the restriction to deviations that have $\hat{x}_{l}=1$ is without loss of generality.

To show that when $\mu<\tilde{\mu}$, our model has no mixed strategy equilibrium, suppose by way of contradiction that it did. Note that the preliminary result established above implies that the high-quality bank strictly prefers the least-cost separating outcome to any other offer in the support of the offer distribution and the low-quality bank strictly prefers any offer greater than $\underline{\pi} v$ to the least-cost separating outcome. Thus, a deviation by one of the buyers to a pure strategy contract close to the least-cost separating outcome, and more profitable when accepted, attracts high-quality banks with probability close to 1 and low-quality banks with probability close to zero.

Suppose now that $\mu \geq \tilde{\mu}$. We will show a mixed strategy equilibrium exists where the distribution over contracts $F(z)$ is given by

$$
F\left(t_{l}\right)=\left(\frac{t_{l}-\underline{\pi} v}{\mu(\bar{\pi}-\underline{\pi}) v}\right)^{\frac{\mu}{d(1-\mu)}-1} .
$$

Since $t_{l} \geq \underline{\pi v}$ and profits are zero at every contract in the support of $F$, it follows that $\hat{x}_{h} \bar{\pi} v \geq \hat{t}_{h}$. Next we show that the best deviations $\hat{z}$ have the property that for some $z$ in the support of $F$, the high-quality bank is indifferent between $z$ and $\hat{z}$, or

$$
\hat{t}_{h}+\left(1-\hat{x}_{h}\right)(\bar{\pi} v-c)=t_{h}+\left(1-x_{h}\right)(\bar{\pi} v-c) .
$$

To see this result, notice that if for all $z$, the left side of (3) is strictly greater than the right side, then $\hat{t}_{h}$ can be reduced and profits can be raised. If the left side of (3) is strictly below the right side for all $z$, then $\delta_{1}(\hat{z}, z ; \bar{\pi})=0$ for all $z$ and profits cannot be strictly positive.

Clearly, the best deviation satisfies the incentive constraint of the low-quality bank with equality, or

$$
\hat{t}_{l}+\left(1-\hat{x}_{l}\right)(\underline{\pi} v-c)=\hat{t}_{h}+\left(1-\hat{x}_{h}\right)(\underline{\pi} v-c),
$$

and $\hat{x}_{l}=1$.

We now evaluate profits for a deviation of the form $\hat{x}_{h}=x_{h}+\varepsilon$ and $\hat{t}_{h}$ and $\hat{t}_{l}$ given by (3) and (4). For any contract $z$ in the support of $F$, the probability of attracting either a highor low-quality bank to contract $z$ is given by $F\left(t_{l}\right)$. Since the high-quality bank is indifferent between $z$ and $\hat{z}$, the probability of attracting the high-quality bank to $\hat{z}$ is given by $F\left(t_{l}\right)$. It is straightforward to show that the deviation contract has $\hat{t}_{l}$ satisfying

$$
\hat{t}_{l}=t_{l}+(\bar{\pi}-\underline{\pi}) v \varepsilon .
$$

Thus, the probability of attracting the low-quality bank to the deviation is given by $F\left(\hat{t}_{l}\right)=$ $F\left(t_{l}+(\bar{\pi}-\underline{\pi}) v \varepsilon\right)$. Holding fixed $\left(x_{h}, t_{h}\right)$, we then define profits from this deviation as a 
function of $\varepsilon$ as

$$
\begin{aligned}
\Pi(\varepsilon)= & (1-\mu) F\left(t_{l}+(\bar{\pi}-\underline{\pi}) v \varepsilon\right)\left(\underline{\pi} v-t_{l}-(\bar{\pi}-\underline{\pi}) v \varepsilon\right) \\
& +\mu F\left(t_{l}\right)\left(\left(x_{h}+\varepsilon\right) \bar{\pi} v-t_{h}-(\bar{\pi} v-c) \varepsilon\right) .
\end{aligned}
$$

Next we prove that profits are globally concave and attain a maximum at $\epsilon=0$. First, note that

$$
\begin{aligned}
\Pi^{\prime}(0) & =(1-\mu)(\bar{\pi}-\underline{\pi}) v f\left(t_{l}\right)\left(\underline{\pi} v-t_{l}\right)-(1-\mu) F\left(t_{l}\right)(\bar{\pi}-\underline{\pi}) v+\mu F\left(t_{l}\right) c \\
& =0 .
\end{aligned}
$$

Suppose now that the distribution $F$ is continuous, has no mass point, has a connected support that is a subset of $[\underline{\pi} v, \hat{p}(\mu)]$, and $F\left(t_{l}\right)<1$ if $t_{l}<\hat{p}(\mu)$. Then solving the differential equation (6), we obtain that it has a unique solution given by (2).

We now prove that $\Pi^{\prime \prime}(\epsilon)<0$ for all $\epsilon$, so $\varepsilon=0$ is a global maximum. Differentiating (5) twice gives

$$
\begin{aligned}
\Pi^{\prime \prime}(\epsilon)= & (1-\mu)((\bar{\pi}-\underline{\pi}) v)^{2} f^{\prime}\left(t_{l}+(\bar{\pi}-\underline{\pi}) v \epsilon\right)\left(\underline{\pi} v-t_{l}-(\bar{\pi}-\underline{\pi}) v \epsilon\right) \\
& -2(1-\mu)((\bar{\pi}-\underline{\pi}) v)^{2} f\left(t_{l}+(\bar{\pi}-\underline{\pi}) v \epsilon\right) .
\end{aligned}
$$

Now, note that

$$
f^{\prime}\left(t_{l}\right)=\frac{f\left(t_{l}\right)}{t_{l}-\underline{\pi} v}\left[\frac{\mu c}{(1-\mu)(\bar{\pi}-\underline{\pi}) v}-2\right] .
$$

Hence,

$$
\begin{aligned}
\Pi^{\prime \prime}(\epsilon)= & (1-\mu)((\bar{\pi}-\underline{\pi}) v)^{2} f\left(t_{l}+(\bar{\pi}-\underline{\pi}) v \epsilon\right) \\
& \times\left[-2+\left(\underline{\pi} v-t_{l}-(\bar{\pi}-\underline{\pi}) v \varepsilon\right)\left(\frac{\mu c}{(1-\mu)(\bar{\pi}-\underline{\pi}) v}-2\right)\left(\frac{1}{t_{l}+(\bar{\pi}-\underline{\pi}) v \varepsilon-\underline{\pi} v}\right)\right] \\
= & -(1-\mu)((\bar{\pi}-\underline{\pi}) v)^{2} f\left(t_{l}++(\bar{\pi}-\underline{\pi}) v \epsilon\right) \frac{\mu c}{(1-\mu)(\bar{\pi}-\underline{\pi}) v} \\
< & 0 .
\end{aligned}
$$

Consider the solution to the differential equation in (6) with the boundary condition that $F(\hat{p}(\mu))=1$. This solution coincides with $(2)$.

Next, we show that the above mixed strategy equilibrium is unique in the class of monotone equilibria. Since the solution to (6) is unique in the class of distributions that are continuous, that have no mass point, that have a connected support which is a subset of $[\underline{\pi} v, \hat{p}(\mu)]$, and that satisfy $F\left(t_{l}\right)<1$ if $t_{l}<\hat{p}(\mu)$, we need only show that any mixed strategy distribution must have these properties.

To see that $t_{l} \leq \hat{p}(\mu)$, let $\hat{x}_{h}\left(t_{l}\right)$ and $\hat{t}_{h}\left(t_{l}\right)$ denote the set of offers $\left(x_{h}, t_{h}\right)$ to the highquality bank implied by a binding incentive constraint for the low-quality bank and zero profits as a function of $t_{l}$. These solutions have the properties that $\hat{x}_{h}\left(t_{l}\right)$ is increasing and $\hat{x}_{h}(\hat{p}(\mu))=1$. Since $\hat{x}_{h}\left(t_{l}\right) \leq 1$, it follows that $t_{l} \leq \hat{p}(\mu)$.

To show that $F$ has no mass points, suppose there exists a $t_{l} \in \operatorname{Supp}(F)$ and that $F\left(t_{l}+\right)>F\left(t_{l}-\right)$ with $t_{l}>\underline{\pi} v$.. Then, consider a deviation by one of the buyers to

$$
z^{\prime}=\left(\hat{x}\left(t_{l}\right)-\varepsilon, \hat{t}_{h}\left(t_{l}\right)-\varepsilon(\bar{\pi} v-c), 1, t_{l}-\varepsilon(\bar{\pi}-\underline{\pi}) v\right) .
$$

Note that by construction the high type is indifferent between $z^{\prime}$ and $\hat{z}\left(t_{l}\right)$ whereas the low type strictly prefers $\hat{z}\left(t_{l}\right)$. Now suppose that $F\left(t_{l}+\right)-F\left(t_{l}-\right)=f\left(t_{l}\right)>0$, that is, $F$ puts 
mass $f\left(t_{l}\right)$ on $t_{l}$. Clearly, the deviation contract $z^{\prime}$ is incentive compatible. Hence, as $\varepsilon$ converges to 0 , the fraction of high-quality banks attracted to $z^{\prime}, \int \delta_{j}\left(z^{\prime}, z_{-j} ; \pi\right) d F\left(z_{-j}\right)$ approaches $F\left(t_{l}-\right)+f\left(t_{l}\right) / 2$ (the $1 / 2$ follows from the uniform tie-breaking rule). The fraction of low-quality banks attracted to $z^{\prime}, \int \delta_{j}\left(z^{\prime}, z_{-j} ; \underline{\pi}\right) d F\left(z_{-j}\right)$ approaches $F\left(t_{l}-\right)$. Hence, the profits for this deviation converge to

$$
\begin{aligned}
& \mu\left(F\left(t_{l}-\right)+f / 2\right)\left(\hat{x}_{h}\left(t_{l}\right) \bar{\pi} v-\hat{t}_{h}\left(t_{l}\right)\right)+(1-\mu) F\left(t_{l}-\right)\left(\underline{\pi} v-t_{l}\right)= \\
& F\left(t_{l}-\right)\left[\mu\left(\hat{x}_{h}\left(t_{l}\right) \bar{\pi} v-\hat{t}_{h}\left(t_{l}\right)\right)+(1-\mu)\left(\underline{\pi} v-t_{l}\right)\right]+\mu f\left(t_{l}\right) / 2\left(\hat{x}_{h}\left(t_{l}\right) \bar{\pi} v-\hat{t}_{h}\left(t_{l}\right)\right)>0,
\end{aligned}
$$

where the inequality follows from the fact that (1) holds with equality and $t_{l}>\underline{\pi} v$. Hence, for $\varepsilon$ sufficiently small enough, $z^{\prime}$ is a profitable deviation that yields the necessary contradiction.

To see that the upper bound of the support of $F$ is $\hat{p}(\mu)$, suppose for some $t_{l}<\hat{p}(\mu)$, $F\left(t_{l}\right)=1$. We prove that there exists a profitable deviation. Consider the deviation $z^{\prime}=$ $(1, \hat{p}(\mu)-\varepsilon, 1, \hat{p}(\mu)-\varepsilon)$. Straightforward algebra can be used to show that

$$
\hat{t}_{h}\left(t_{l}\right)+\left(1-\hat{x}_{h}\left(t_{l}\right)\right)(\bar{\pi} v-c)=\hat{x}_{h}\left(t_{l}\right) \hat{p}(\mu)+\left(1-\hat{x}_{h}\left(t_{l}\right)\right)(\bar{\pi} v-\mu c) .
$$

Since $t_{l}<\hat{p}(\mu), \hat{x}_{h}\left(t_{l}\right)<1$ and $\mu>\tilde{\mu}$, it follows that $\hat{p}(\mu)>\bar{\pi} v-\mu c$, so that

$$
\hat{p}(\mu)>\hat{t}_{h}\left(t_{l}\right)+\left(1-\hat{x}_{h}\left(t_{l}\right)\right)(\bar{\pi} v-c) .
$$

Hence, for $\varepsilon>0$ and sufficiently small, the deviation contract $z^{\prime}$ attracts both high- and low-quality banks with probability 1 against the equilibrium, or $\int \delta_{j}\left(z^{\prime} ; z_{-j} ; \pi\right) d F\left(z_{-j}\right)=1$ for $\pi=\underline{\pi}, \bar{\pi}$. But profits at $z^{\prime}=\varepsilon>0$, which implies that a profitable deviation would exist. Thus, for all $t_{l}<\hat{p}(\mu), F\left(t_{l}\right)<1$.

Suppose next that $F\left(t_{l}\right)$ does not have connected support. That is, suppose there exists an interval $\left[t_{1}, t_{2}\right]$ such that $F\left(t_{l}\right)=F\left(t_{1}\right), \forall t_{l} \in\left[t_{1}, t_{2}\right]$. Since $F\left(t_{1}\right)<1$ and for all $t<\hat{p}(\mu), F(t)<1$, it must be that $t_{2}<\hat{p}(\mu)$. Consider then a deviation of the form $z^{\prime}=\left(\hat{x}_{h}\left(t_{2}\right), \hat{t}_{h}\left(t_{2}\right)-\varepsilon, 1, t_{2}\right)$. Since $t_{2}<\hat{p}(\mu)$ the incentive constraint for the high-quality bank is slack at $\hat{z}\left(t_{2}\right)$, so that if $\varepsilon>0$ is chosen sufficiently small enough, $z^{\prime}$ is incentive compatible. Furthermore, for $\varepsilon$ sufficiently small the fraction of high- and low-quality banks attracted to $z^{\prime}$ is the same as $\hat{z}\left(t_{2}\right)$, since $F\left(t_{l}\right)$ is constant in a neighborhood below $t_{2}$. This implies that $z^{\prime}$ makes higher profit than $\hat{z}\left(t_{2}\right)$ and hence $z^{\prime}$ is a profitable deviation.

Since the distribution has the desired properties, it must coincide with the distribution $F(\cdot)$ in $(2)$.

Q.E.D.

\section{Basic Properties of Partial Pooling Allocations}

Lemma 1 Consider the partial pooling allocation described in section II.B with $x_{l}=1$. Then we have the following:

1. If the incentive constraints in the two-period model are satisfied and equilibrium profits are nonnegative, participation constraint for the high-quality bank and the constraint imposed by Bertrand competition can be respectively simplified to $x_{h} \leq \bar{x}_{h}\left(\mu_{h}, v\right)$ and 
$x_{h} \geq \underline{x}_{h}\left(\mu_{h}, v\right)$, where $\bar{x}_{h}\left(\mu_{h}, v\right)$ and $\underline{x}_{h}\left(\mu_{h}, v\right)$ are given by

$$
\begin{aligned}
& x_{h} \leq \bar{x}\left(\mu_{h}^{\prime}, v\right)=\frac{\beta\left[\Delta\left(\mu_{h}^{\prime} ; \bar{\pi}\right)-\Delta\left(\mu_{h}^{\prime} ; \underline{\pi}\right)\left(1-\frac{\mu}{\mu_{h}^{\prime}}\right)\right]+\left(1-\frac{\mu}{\mu_{h}^{\prime}}\right) c}{\bar{\pi} v-c-\hat{p}(\mu)+\left(1-\frac{\mu}{\mu_{h}^{\prime}}\right) c} \\
& \frac{\frac{2-\mu-\mu_{h}^{\prime}}{\mu_{h}^{\prime}(1-\mu)}}{(\bar{\pi}-\underline{\pi}) v+c \frac{2-\mu-\mu_{h}^{\prime}}{\mu_{h}^{\prime}(1-\mu)}}\left(c-\beta \Delta\left(\mu_{h}^{\prime} ; \underline{\pi}\right)\right)=\underline{x}\left(\mu_{h}^{\prime}, v\right) \leq x_{h} .
\end{aligned}
$$

2. The upper bound implied by the participation constraint

$$
t_{h}+\left(1-x_{h}\right)(\bar{\pi} v-c)+\beta V\left(\mu_{h}^{\prime} ; \bar{\pi}\right) \geq \bar{\pi} v-c+\beta V(0 ; \bar{\pi})
$$

is decreasing in $\mu_{h}^{\prime}$ when $\mu_{h}^{\prime} \geq \tilde{\mu}$ and increasing otherwise.

3. The lower bound $\underline{x}_{h}$, when positive, is decreasing in $\mu_{h}^{\prime}$.

4. Aggregate volume implied by the upper bound in (9) is maximized at $\mu_{h}^{\prime}=\tilde{\mu}$.

5. Ex ante welfare implied by the upper bound in (9) is maximized at $\mu_{h}^{\prime}=\tilde{\mu}$.

6. When $\mu_{h}^{\prime}$ is large enough, $\underline{x}_{h} \leq \bar{x}_{h}$.

\section{Proof.}

1. Using the incentive constraints and zero profits, we solve for $t_{h}$ and $t_{l}$ to obtain

$$
\begin{aligned}
t_{h} & =\left(\hat{p}(\mu)+c\left(\frac{\mu}{\mu_{h}^{\prime}}-1\right)\right) x_{h}+\left(\frac{\mu}{\mu_{h}^{\prime}}-1\right)\left(\beta \Delta\left(\mu_{h}^{\prime} ; \underline{\pi}\right)-c\right) \\
t_{l} & =\underline{\pi} v+\frac{\mu}{\mu_{h}^{\prime}}\left(\mu_{h}^{\prime}(\bar{\pi}-\underline{\pi}) v+c\right) x_{h}+\frac{\mu}{\mu_{h}^{\prime}}\left(\beta \Delta\left(\mu_{h}^{\prime} ; \underline{\pi}\right)-c\right) .
\end{aligned}
$$

Substituting for $t_{h}$ in (9), we get

$$
\begin{aligned}
& \left(\hat{p}(\mu)+c\left(\frac{\mu}{\mu_{h}^{\prime}}-1\right)\right) x_{h}+\left(\frac{\mu}{\mu_{h}^{\prime}}-1\right)\left(\beta \Delta\left(\mu_{h}^{\prime} ; \underline{\pi}\right)-c\right)-x_{h}(\bar{\pi} v-c)+\beta \Delta\left(\mu_{h}^{\prime} ; \bar{\pi}\right) \geq 0 \\
& \left(\frac{\mu}{\mu_{h}^{\prime}}-1\right)\left(\beta \Delta\left(\mu_{h}^{\prime} ; \underline{\pi}\right)-c\right)+\beta \Delta\left(\mu_{h}^{\prime} ; \bar{\pi}\right) \geq\left[\bar{\pi} v-c-\left(\hat{p}(\mu)+c\left(\frac{\mu}{\mu_{h}^{\prime}}-1\right)\right)\right] x_{h} .
\end{aligned}
$$

Note that since $\mu \leq \mu^{*}$ and that $\mu \leq \mu_{h}^{\prime}, \bar{\pi} v-c-\hat{p}(\mu) \geq 0$ and $-c\left(\mu / \mu_{h}^{\prime}-1\right) \geq 0$. Hence, we have

$$
\bar{x}_{h}=\frac{\left(\frac{\mu}{\mu_{h}^{\prime}}-1\right)\left(\beta \Delta\left(\mu_{h}^{\prime} ; \underline{\pi}\right)-c\right)+\beta \Delta\left(\mu_{h}^{\prime} ; \bar{\pi}\right)}{\bar{\pi} v-c-\left(\hat{p}(\mu)+c\left(\frac{\mu}{\mu_{h}^{\prime}}-1\right)\right)} \geq x_{h} .
$$

Next, substituting for $t_{h}$ and $t_{l}$ from (10) into the constraint implied by Bertrand competition, we have that

$$
\begin{gathered}
\frac{1}{2} \mu\left[(1-\mu)(\bar{\pi}-\underline{\pi}) v x_{h}+c\left(1-\frac{\mu}{\mu_{h}^{\prime}}\right) x_{h}+\left(1-\frac{\mu}{\mu_{h}^{\prime}}\right)\left(\beta \Delta\left(\mu_{h}^{\prime} ; \underline{\pi}\right)-c\right)\right] \leq \\
(1-\mu)\left[\frac{\mu}{\mu_{h}^{\prime}}\left(\mu_{h}^{\prime}(\bar{\pi}-\underline{\pi}) v+c\right) x_{h}+\frac{\mu}{\mu_{h}^{\prime}}\left(\beta \Delta\left(\mu_{h}^{\prime} ; \underline{\pi}\right)-c\right)\right] .
\end{gathered}
$$


After simplification, this inequality can be written as

$$
\frac{2-\mu-\mu_{h}^{\prime}}{\mu_{h}^{\prime}}\left(c-\beta \Delta\left(\mu_{h}^{\prime} ; \underline{\pi}\right)\right) \leq x_{h}\left[(1-\mu)(\bar{\pi}-\underline{\pi}) v+\frac{2-\mu-\mu_{h}^{\prime}}{\mu_{h}^{\prime}} c\right] .
$$

The right side of this inequality is positive, so that dividing both sides by the expression in brackets on the right side, we obtain the expression for the lower bound:

$$
\underline{x_{h}}=\frac{\frac{2-\mu-\mu_{h}^{\prime}}{\mu_{h}^{\prime}(1-\mu)}\left(c-\beta \Delta\left(\mu_{h}^{\prime} ; \underline{\pi}\right)\right)}{(\bar{\pi}-\underline{\pi}) v+\frac{2-\mu-\mu_{h}^{\prime}}{\mu_{h}^{\prime}(1-\mu)} c} \leq x_{h} .
$$

2. Suppose that $\mu_{h}^{\prime} \geq \tilde{\mu}$. The denominator of (11) is an increasing function of $\mu_{h}^{\prime}$. Hence, it suffices to show that the numerator is decreasing in $\mu_{h}^{\prime}$. Using the definition of the value function $V(\cdot, \cdot)$, we can write the numerator as

$$
\begin{aligned}
& \left(\frac{\mu}{\mu_{h}^{\prime}}-1\right)\left(\beta \frac{\mu_{h}^{\prime}-\tilde{\mu}}{1-\tilde{\mu}}(\bar{\pi}-\underline{\pi}) v-c\right)+\beta \frac{\left(\mu_{h}^{\prime}-\tilde{\mu}\right)^{2}}{\mu_{h}^{\prime}(1-\tilde{\mu})}(\bar{\pi}-\underline{\pi}) v= \\
& \beta \frac{\left(\mu_{h}^{\prime}-\tilde{\mu}\right)}{\mu_{h}^{\prime}(1-\tilde{\mu})}(\bar{\pi}-\underline{\pi}) v\left[\mu_{h}^{\prime}-\tilde{\mu}+\mu-\mu_{h}^{\prime}\right]+c\left(1-\frac{\mu}{\mu_{h}^{\prime}}\right)= \\
& \beta \frac{(\bar{\pi}-\underline{\pi}) v(\mu-\tilde{\mu})}{1-\tilde{\mu}}\left[1-\frac{\tilde{\mu}}{\mu_{h}^{\prime}}\right]+c\left(1-\frac{\mu}{\mu_{h}^{\prime}}\right) .
\end{aligned}
$$

Using the property that $\tilde{\mu} /(1-\tilde{\mu})=(\bar{\pi}-\underline{\pi}) v / c$, we obtain that the derivative of the last expression with respect to $\mu_{h}^{\prime}$ is given by

$$
-\frac{c}{\left(\mu_{h}^{\prime}\right)^{2}}\left[\beta\left(\frac{(\bar{\pi}-\underline{\pi}) v}{c}\right)^{2}(\tilde{\mu}-\mu)-\mu\right] .
$$

Using the definition of the adverse selection discount, $d$, we can write this expression as

$$
-\frac{c}{\left(\mu_{h}^{\prime}\right)^{2}}\left[\beta d^{2}\left(\frac{d}{1+d}-\mu\right)-\mu\right] \text {. }
$$

Here, we show that the term in brackets is positive and hence the derivative of the numerator is negative:

$$
\begin{aligned}
\beta d^{2}\left(\frac{d}{1+d}-\mu\right)-\mu & \geq(1+d) d\left(\frac{d}{1+d}-\mu\right)-\mu \\
& \geq d^{2}-\left(1-\frac{1}{d}\right)\left(d^{2}+d+1\right) \\
& =\frac{1}{d^{3}} \geq 0,
\end{aligned}
$$

where the first inequality follows from the assumption that banks are sufficiently patient and the second inequality follows from $\mu \leq \mu^{*}=1-1 / d$.

To see that $\bar{x}_{h}$ is increasing in $\mu_{h}^{\prime}$ when $\mu_{h}^{\prime} \leq \tilde{\mu}$, note that when $\mu_{h}^{\prime} \leq \tilde{\mu}, \Delta\left(\mu_{h}^{\prime} ; \pi\right)=0$, so that we can write the upper bound as

$$
\bar{x}_{h}=\frac{c}{\frac{\bar{\pi} v-c-\hat{p}(\mu)}{1-\mu / \mu_{h}^{\prime}}+c} .
$$

Clearly this function is increasing in $\mu_{h}^{\prime}$. 
3. Suppose that $\underline{x}_{h}$ is positive. Note that the expression $c-\beta \Delta\left(\mu_{h}^{\prime} ; \underline{\pi}\right)$ is decreasing in $\mu_{h}^{\prime}$. In addition,

$$
\frac{\frac{2-\mu-\mu_{h}^{\prime}}{\mu_{h}^{\prime}(1-\mu)}}{(\bar{\pi}-\underline{\pi}) v+\frac{2-\mu-\mu_{h}^{\prime}}{\mu_{h}^{\prime}(1-\mu)} c}=\frac{1}{c+\frac{(\bar{\pi}-\underline{\pi}) v}{\frac{2-\mu-\mu_{h}^{\prime}}{\mu_{h}^{\prime}(1-\mu)}}}
$$

The function

$$
\frac{2-\mu-\mu_{h}^{\prime}}{\mu_{h}^{\prime}(1-\mu)}=\frac{1}{\mu_{h}^{\prime}}+\frac{1}{\mu_{h}^{\prime}(1-\mu)}-\frac{1}{1-\mu}
$$

is decreasing in $\mu_{h}^{\prime}$ and hence the expression in (14) is decreasing in $\mu_{h}^{\prime}$. Thus, $\underline{x}_{h}$ is the product of two decreasing functions. Since both are positive functions, $\underline{x}_{h}$ must be decreasing.

4 and 5 . Note that aggregate welfare is given by

$$
\hat{p}(\mu)-(1-T) c+\beta \mu V\left(\mu_{h}^{\prime} ; \bar{\pi}\right)+\beta\left(\frac{\mu}{\mu_{h}^{\prime}}-\mu\right) V\left(\mu_{h}^{\prime} ; \underline{\pi}\right)+\beta\left(1-\frac{\mu}{\mu_{h}^{\prime}}\right) V(0 ; \underline{\pi}),
$$

where $T$ is aggregate volume. To prove our claims, we first obtain a simplified version of the continuation value

$$
\beta \mu V\left(\mu_{h}^{\prime} ; \bar{\pi}\right)+\beta\left(\frac{\mu}{\mu_{h}^{\prime}}-\mu\right) V\left(\mu_{h}^{\prime} ; \underline{\pi}\right)+\beta\left(1-\frac{\mu}{\mu_{h}^{\prime}}\right) V(0 ; \underline{\pi})
$$

and show for later use that this continuation value is increasing in $\mu_{h}^{\prime}$. Note that when $\mu_{h}^{\prime} \leq \tilde{\mu}$, the continuation value is constant since $V\left(\mu_{h}^{\prime} ; \pi\right)=V(0 ; \pi)$ and hence it is weakly increasing. For values of $\mu_{h}^{\prime} \geq \tilde{\mu}$, the continuation value is given by

$$
\begin{aligned}
& \beta \mu\left[V\left(\mu_{h}^{\prime} ; \bar{\pi}\right)-V(0 ; \bar{\pi})\right]+\beta\left(\frac{\mu}{\mu_{h}^{\prime}}-\mu\right)\left[V\left(\mu_{h}^{\prime} ; \underline{\pi}\right)-V(0 ; \underline{\pi})\right]+\beta(1-\mu) V(0 ; \underline{\pi})+\beta \mu V(0 ; \bar{\pi})= \\
& \beta \mu(\bar{\pi}-\underline{\pi}) v \frac{\left(\mu_{h}^{\prime}-\tilde{\mu}\right)^{2}}{(1-\tilde{\mu}) \mu_{h}^{\prime}}+\beta\left(\frac{\mu}{\mu_{h}^{\prime}}-\mu\right)(\bar{\pi}-\underline{\pi}) v \frac{\mu_{h}^{\prime}-\tilde{\mu}}{1-\tilde{\mu}}+\beta(1-\mu) V(0 ; \underline{\pi})+\beta \mu V(0 ; \bar{\pi})= \\
& \beta \frac{\mu_{h}^{\prime}-\tilde{\mu}}{1-\tilde{\mu}}(\bar{\pi}-\underline{\pi}) v\left[\mu \frac{\mu_{h}^{\prime}-\tilde{\mu}}{\mu_{h}^{\prime}}+\frac{\mu}{\mu_{h}^{\prime}}-\mu\right]+\beta(1-\mu) V(0 ; \underline{\pi})+\beta \mu V(0 ; \bar{\pi})= \\
& \beta \frac{\mu_{h}^{\prime}-\tilde{\mu}}{1-\tilde{\mu}}(\bar{\pi}-\underline{\pi}) v\left[\mu \frac{1-\tilde{\mu}}{\mu_{h}^{\prime}}\right]+\beta(1-\mu) V(0 ; \underline{\pi})+\beta \mu V(0 ; \bar{\pi})= \\
& \beta \frac{\mu_{h}^{\prime}-\tilde{\mu}}{\mu_{h}^{\prime}}(\bar{\pi}-\underline{\pi}) v \mu+\beta(1-\mu) V(0 ; \underline{\pi})+\beta \mu V(0 ; \bar{\pi}) .
\end{aligned}
$$

The above function is increasing in $\mu_{h}^{\prime}$.

In what follows, we first show that ex ante welfare in (15) is maximized at $\mu_{h}^{\prime}=\tilde{\mu}$. This establishes claim 5. In addition, we show that aggregate volume $T$ is increasing in $\mu_{h}^{\prime} \leq \tilde{\mu}$. Since continuation values are increasing in $\mu_{h}^{\prime}$, that welfare is maximized at $\mu_{h}^{\prime}=\tilde{\mu}$ implies that volume is also maximized at $\mu_{h}^{\prime}=\tilde{\mu}$.

Before studying properties of welfare, note that if we set $\mu_{h}^{\prime}=1$, given that banks are sufficiently patient, an argument similar to that of proposition 4 shows that $\bar{x}_{h}$ must be negative. Hence, in order to have a partial pooling allocation, $\mu_{h}^{\prime}$ must be bounded above. In other words, admissible values of $\mu_{h}^{\prime}$ belong to the interval $[\mu, \bar{\mu}]$, where $\bar{\mu}$ is such that when we set $\mu_{h}^{\prime}=\bar{\mu}, \bar{x}_{h}$ is equal to 0 . 
To study ex ante welfare, suppose that $\mu_{h}^{\prime} \geq \tilde{\mu}$ and recall that total volume is given by

$$
T=\frac{\mu}{\mu_{h}^{\prime}}\left(\bar{x}_{h}-1\right)+1
$$

and hence, ex ante welfare can be written as

$$
\kappa+\frac{\mu}{\mu_{h}^{\prime}}\left(\bar{x}_{h}-1\right) c+\beta \frac{\mu_{h}^{\prime}-\tilde{\mu}}{\mu_{h}^{\prime}}(\bar{\pi}-\underline{\pi}) v \mu,
$$

where $\kappa$ is independent of $\mu_{h}^{\prime}$ and we have used (16) to rewrite the continuation utilities. We can rewrite the above as

$$
\kappa^{\prime}+c \frac{\mu}{\mu_{h}^{\prime}}\left(\bar{x}_{h}-1-\frac{\tilde{\mu}(\bar{\pi}-\underline{\pi}) v}{c}\right)=\kappa^{\prime}+c \frac{\mu}{\mu_{h}^{\prime}}\left(\bar{x}_{h}-1-\beta \frac{d^{2}}{1+d}\right),
$$

where $\kappa^{\prime}$ is independent of $\mu_{h}^{\prime}$. As we have shown in the proof of property 2 , specifically in (13), both the numerator and the denominator of $\bar{x}_{h}$ are linear functions of $y=\frac{1}{\mu_{h}^{\prime}}$. This implies that $\bar{x}_{h}-1-\beta d^{2} /(1+d)$ has the same property. That is, $a, a^{\prime}, b, b^{\prime}$ exists such that

$$
\bar{x}_{h}-1-\frac{\beta d^{2}}{1+d}=\frac{-a+a^{\prime} \frac{1}{\bar{\mu}_{h}^{\prime}}}{b-b^{\prime} \frac{1}{\mu_{h}^{\prime}}}=\frac{-a+a^{\prime} y}{b-b^{\prime} y},
$$

where $b=\bar{\pi} v-\hat{p}(\mu)$ is positive. Note that $\bar{x}_{h}$ is a decreasing function of $\mu_{h}^{\prime}$ and hence an increasing function of $y$. This implies that the derivative of the above expression with respect to $y$ is positive, or

$$
\frac{b^{\prime} a-a^{\prime} b}{\left(b-b^{\prime} y\right)^{2}} \geq 0
$$

Now, if we let (17) as a function $y$ be called $f(y)$, then we can write

$$
f(y)=\kappa^{\prime}+\mu y \frac{-a+a^{\prime} y}{b-b^{\prime} y}
$$

and we have

$$
\begin{aligned}
f^{\prime}(y) & =\mu \frac{-a+a^{\prime} y}{b-b^{\prime} y}+\mu y \frac{b^{\prime} a-a^{\prime} b}{\left(b-b^{\prime} y\right)^{2}} \\
f^{\prime \prime}(y) & =\mu \frac{b^{\prime} a-a^{\prime} b}{\left(b-b^{\prime} y\right)^{2}}+\mu \frac{b^{\prime} a-a^{\prime} b}{\left(b-b^{\prime} y\right)^{2}}+2 \mu y \frac{\left(b^{\prime} a-a^{\prime} b\right) b^{\prime}}{\left(b-b^{\prime} y\right)^{3}} \\
& =2 \mu \frac{\left(b^{\prime} a-a^{\prime} b\right)\left(b-b^{\prime} y\right)+y\left(b^{\prime} a-a^{\prime} b\right) b^{\prime}}{\left(b-b^{\prime} y\right)^{3}} \\
& =2 \mu b \frac{b^{\prime} a-a^{\prime} b}{\left(b-b^{\prime} y\right)^{3}} .
\end{aligned}
$$

Note that from (18) and the fact that $b>0$, the above expression is convex. This implies that ex ante welfare as a function of $\mu_{h}^{\prime}$, given by $f\left(\frac{1}{\mu_{h}^{\prime}}\right)$ is convex since both $f(\cdot)$ and $\frac{1}{\mu_{h}^{\prime}}$ are convex functions. Hence, in order to show that welfare is maximized at $\mu_{h}^{\prime}=\tilde{\mu}$, it is sufficient to show that $f\left(\frac{1}{\tilde{\mu}}\right) \geq f\left(\frac{1}{\bar{\mu}}\right)$, where $\bar{\mu}$ is such that $\bar{x}_{h}=0$. Extensive and cumbersome algebra (available upon request) establishes that since banks are sufficiently patient, this inequality is satisfied. Hence, among values of $\mu_{h}^{\prime}$ for which $\bar{x}_{h} \geq 0$, welfare 
is maximized at $\mu_{h}^{\prime}=\tilde{\mu}$. As we have shown before, when $\mu_{h}^{\prime} \leq \tilde{\mu}, \bar{x}_{h}$ is increasing in $\mu_{h}^{\prime}$. This implies that aggregate volume is increasing in $\mu_{h}^{\prime}$ for values of $\mu_{h}^{\prime} \leq \tilde{\mu}$ and hence so is welfare. This establishes the claim that welfare is maximized at $\mu_{h}^{\prime}=\tilde{\mu}$.

Finally, note that the above establishes that for values of $\mu_{h}^{\prime} \geq \tilde{\mu}$, volume is maximized at $\mu_{h}^{\prime}=\tilde{\mu}$. Furthermore, as we just showed, volume is increasing in $\mu_{h}^{\prime}$ when $\mu_{h}^{\prime} \leq \tilde{\mu}$. Hence, volume is maximized at $\mu_{h}^{\prime}=\tilde{\mu}$.

6. In order to prove the claim, let $\mu_{h}^{\prime}$ be such that $\Delta\left(\mu_{h}^{\prime} ; \underline{\pi}\right)=c$. This is the value of $\mu_{h}^{\prime}$ for which $\underline{x}_{h}=0$. At this value, $\bar{x}_{h}$ is given by

$$
\begin{aligned}
\bar{x}_{h} & =\frac{\left(\frac{\mu}{\mu_{h}^{\prime}}-1\right)\left(\beta \Delta\left(\mu_{h}^{\prime} ; \underline{\pi}\right)-c\right)+\beta \Delta\left(\mu_{h}^{\prime} ; \bar{\pi}\right)}{\bar{\pi} v-c-\left(\hat{p}(\mu)+c\left(\frac{\mu}{\mu_{h}^{\prime}}-1\right)\right)} \\
& =\frac{\beta \Delta\left(\mu_{h}^{\prime} ; \bar{\pi}\right)}{\bar{\pi} v-c-\left(\hat{p}(\mu)+c\left(\frac{\mu}{\mu_{h}^{\prime}}-1\right)\right)}>0 .
\end{aligned}
$$

This establishes the claim.

Q.E.D.

\subsection{Establishing the constraint (A13)}

We establish the result by showing that $\bar{x}_{h}\left(\mu_{h}^{\prime}, v\right)$ is less than the right side of (A13). Using (13), we have that

$$
\bar{x}_{h}\left(\mu_{h}^{\prime}, v\right)=\frac{\beta \frac{\left(\bar{\pi}-\frac{\pi}{\pi}\right) v(\mu-\tilde{\mu})}{1-\tilde{\mu}}\left[1-\frac{\tilde{\mu}}{\mu_{h}^{\prime}}\right]+c\left(1-\frac{\mu}{\mu_{h}^{\prime}}\right)}{\bar{\pi} v-c-\left(\hat{p}(\mu)+c\left(\frac{\mu}{\mu_{h}^{\prime}}-1\right)\right)},
$$

so that using the definition of $\mu^{*}$ and simplifying, we have that

$$
\bar{x}_{h}\left(\mu_{h}^{\prime}, v\right)=\frac{\beta(\mu-\tilde{\mu}) \frac{\mu^{\prime}-\tilde{\mu}}{(1-\tilde{\mu})}(\bar{\pi}-\underline{\pi}) v+\left(\mu^{\prime}-\mu\right) c}{\mu^{\prime}\left(\mu^{*}-\mu\right)(\bar{\pi}-\underline{\pi}) v+\left(\mu^{\prime}-\mu\right) c} .
$$

Further algebra shows that $\bar{x}_{h}\left(\mu_{h}^{\prime}, v\right)$ is increasing in $\mu$. Since the right side of (A13) equals $\beta \frac{1-\mu_{h}^{\prime}}{1-\tilde{\mu}}$, it is sufficient to show that the following inequality holds:

$$
\frac{\beta\left(\mu^{*}-\tilde{\mu}\right) \frac{\mu^{\prime}-\tilde{\mu}}{(1-\tilde{\mu})}(\bar{\pi}-\underline{\pi}) v+\left(\mu^{\prime}-\mu^{*}\right) c}{\left(\mu^{\prime}-\mu^{*}\right) c} \leq \beta \frac{1-\mu^{\prime}}{1-\tilde{\mu}} .
$$

Straightforward algebra shows that the left side of (19) is concave and decreasing in $\mu^{\prime}$ (all derivations are available upon request). Since the right side of (19) is linear in $\mu^{\prime}$, it is sufficient to show that the above inequality is satisfied at $\mu^{\prime}=\tilde{\mu}$ (the lowest possible value of $\mu^{\prime}$ ) and the derivative of the left side is also lower than the derivative of the right side. This result implies that the inequality in (19) holds for all values of $\mu^{\prime} \geq \tilde{\mu}$. The value of the left side at $\mu^{\prime}=\tilde{\mu}$ is given by 1 , whereas the value of the right side is given by $\beta$. By the assumption that banks are sufficiently patient, $\beta \geq 1$. The derivative of the left side is given by

$$
-\beta \frac{(\bar{\pi}-\underline{\pi}) v}{c} \frac{1}{1-\tilde{\mu}} \frac{\left(\tilde{\mu}-\mu^{*}\right)^{2}}{\left(\mu^{\prime}-\mu^{*}\right)^{2}} .
$$


Evaluating the derivatives of the left and right sides of (19) at $\mu^{\prime}=\tilde{\mu}$, we obtain the following needed inequality:

$$
-\frac{\beta}{1-\tilde{\mu}} \frac{(\bar{\pi}-\underline{\pi}) v}{c} \leq-\frac{\beta}{1-\tilde{\mu}},
$$

which holds under our assumption that $(\bar{\pi}-\underline{\pi}) v / c=d>1$.

Q.E.D.

\section{$5 \quad$ Infinite Horizon Model with Divisible Assets}

In this section, we show that the equilibrium constructed in section IV is also an equilibrium if we allow for trade of a fraction of bank portfolios. To do so, we make the following additional assumption:

$$
\frac{\beta(1-\lambda)}{1-\beta(1-\lambda)} \geq \frac{(\bar{\pi}-\underline{\pi}) v_{\max }^{2}}{c E v}
$$

As in the indivisible asset case, we investigate whether the following is an equilibrium:

$$
\begin{aligned}
& \text { if } \mu_{t}<\min \left\{\mu^{*}\left(v_{t}\right), \mu_{h}\right\},\left(x_{h}, t_{h}\right)=0,\left(x_{l}, t_{l}\right)=\left(1, \underline{\pi} v_{t}\right) \\
& \text { if } \mu_{t} \geq \mu^{*}\left(v_{t}\right),\left(x_{h}, t_{h}\right)=\left(x_{l}, t_{l}\right)=\left(1, \hat{p}\left(\mu_{t}\right) v_{t}\right) \\
& \text { if } \mu_{t} \in\left(\mu_{h}, \mu^{*}(v)\right),\left(x_{h}, t_{h}\right)=\left(x_{l}, t_{l}\right)=(0,0),
\end{aligned}
$$

where $\hat{p}\left(\mu_{t}\right)$ with a little abuse of notation is given by $\bar{\pi} \mu_{t}+\underline{\pi}\left(1-\mu_{t}\right)$ and $\mu^{*}(v)=1-\frac{c}{(\bar{\pi}-\pi) v}$. Since the allocations are identical, the value function is identical as well. We let the beliefs be

$$
\mu^{\prime}\left(x, t ; v_{t}, \mu_{t}\right)=\left\{\begin{array}{cc}
0 & t-x\left(\bar{\pi} v_{t}-c\right) \leq \max \left\{\hat{x} \hat{p}\left(\mu_{t}\right) v_{t}-\hat{x}\left(\bar{\pi} v_{t}-c\right), t_{h}-x_{h}\left(\bar{\pi} v_{t}-c\right)\right\} \\
1 & \text { o.w. }
\end{array}\right.
$$

In what follows, we check that the above strategies as well as the beliefs constitute an equilibrium.

1. We start from the case where $\mu^{*}\left(v_{t}\right) \leq \mu_{h}$. Suppose that $\mu<\mu^{*}(v)$. Consider some deviation, $z^{\prime}=\left(x_{h}^{\prime}, t_{h}^{\prime}, x_{l}^{\prime}, t_{l}^{\prime}\right)$. As in the proof of lemmas 1 and 2 , both of the contracts cannot be preferred statically to $(0,0)$ by the high-quality bank. Suppose $\left(x_{h}^{\prime}, t_{h}^{\prime}\right)$ is statically preferred to $(0,0)$ by the high-quality bank. We first show that this contract must also attract the low type. To see this, note that we must have

$$
\begin{gathered}
t_{h}^{\prime}+\left(1-x_{h}^{\prime}\right)(\bar{\pi} v-c)+\beta(1-\lambda) V(1 ; \bar{\pi}) \geq \bar{\pi} v-c+\beta(1-\lambda) V\left(\mu_{h} ; \bar{\pi}\right) \\
\beta(1-\lambda)\left(\frac{\bar{\pi} E v}{1-\beta(1-\lambda)}-V\left(\mu_{h} ; \bar{\pi}\right)\right) \geq x_{h}^{\prime}(\bar{\pi} v-c)-t_{h}^{\prime} \\
\beta(1-\lambda)\left(\frac{\bar{\pi} E v}{1-\beta(1-\lambda)}-V\left(\mu_{h} ; \underline{\pi}\right)\right)>\beta(1-\lambda)\left(\frac{\bar{\pi} E v}{1-\beta(1-\lambda)}-V\left(\mu_{h} ; \bar{\pi}\right)\right) \\
\geq x_{h}^{\prime}(\bar{\pi} v-c)-t_{h}^{\prime}>x_{h}^{\prime}(\underline{\pi} v-c)-t_{h}^{\prime},
\end{gathered}
$$

which implies that the low-quality bank also prefers $\left(x_{h}^{\prime}, t_{h}^{\prime}\right)$ to $(0,0)$. As in the proof of lemmas 1 and 2, we cannot have $\mu^{\prime}\left(x_{l}^{\prime}, t_{l}^{\prime}\right)=\mu^{\prime}\left(x_{l}^{\prime}, t_{l}^{\prime}\right)=1$. In addition, if $\mu^{\prime}\left(x_{l}^{\prime}, t_{l}^{\prime}\right)=0$, we 
can assume that $x_{l}^{\prime}=1$ and we must have

$$
\begin{aligned}
t_{l}^{\prime}+\beta(1-\lambda) V(0 ; \underline{\pi}) & \geq t_{h}^{\prime}+\left(1-x_{h}^{\prime}\right)(\underline{\pi} v-c)+\beta(1-\lambda) V(1 ; \underline{\pi}) \\
t_{l}^{\prime} & >t_{h}^{\prime}+\left(1-x_{h}^{\prime}\right)(\underline{\pi} v-c)+c .
\end{aligned}
$$

So the profits from this deviation are given by

$$
\begin{aligned}
\mu\left(x_{h}^{\prime} \bar{\pi} v-t_{h}^{\prime}\right)+(1-\mu)\left(\underline{\pi} v-t_{l}^{\prime}\right) & \geq \mu\left(x_{h}^{\prime} \bar{\pi} v-t_{h}^{\prime}\right)+(1-\mu)\left(\underline{\pi} v-t_{h}^{\prime}-\left(1-x_{h}^{\prime}\right)(\underline{\pi} v-c)-c\right) \\
& =\mu\left(x_{h}^{\prime} \bar{\pi} v-t_{h}^{\prime}\right)+(1-\mu)\left(x_{h}^{\prime}(\underline{\pi} v-c)-t_{h}^{\prime}\right) \\
& =x_{h}^{\prime} \hat{p}(\mu) v-t_{h}^{\prime}-c(1-\mu) x_{h}^{\prime} .
\end{aligned}
$$

Since $\mu^{*}(v)>\mu$ and $\left(x_{h}^{\prime}, t_{h}^{\prime}\right)$ is statically preferred to $(0,0)$ by the high-quality bank, it must be that $\hat{p}(\mu) x_{h}^{\prime} \leq t_{h}^{\prime}$, and hence the above expression is negative. Now, suppose that $\mu \geq \mu^{*}(v)$. As we have done before, it is sufficient to show that any contract that attracts the high-quality bank also attracts the low-quality bank. To do this, consider the offer $\left(x^{\prime}, t^{\prime}\right)$ that gives the lowest static payoff to the bank of low quality and is statically preferred to $(1, \hat{p}(\mu))$ by the high-quality bank. Such an offer satisfies

$$
\begin{aligned}
t^{\prime} & =\bar{\pi} v x^{\prime} \\
x^{\prime} \bar{\pi} v+\left(1-x^{\prime}\right)(\bar{\pi} v-c) & =\hat{p}(\mu) v \\
x^{\prime} c & =c-(1-\mu)(\bar{\pi}-\underline{\pi}) v .
\end{aligned}
$$

It is sufficient to show that the low-quality bank prefers this offer to the pooling outcome:

$$
\begin{aligned}
x^{\prime} \bar{\pi} v+\left(1-x^{\prime}\right)(\underline{\pi} v-c)+\beta V(1 ; \underline{\pi}) & \geq \mu(\bar{\pi}-\underline{\pi}) v+c+\beta V(\mu ; \underline{\pi}) \\
{\left[1-(1-\mu) \frac{(\bar{\pi}-\underline{\pi}) v}{c}\right]((\bar{\pi}-\underline{\pi}) v+c) } & \geq \mu(\bar{\pi}-\underline{\pi}) v+c+\beta V(\mu ; \underline{\pi})-\frac{\beta}{1-\beta} \bar{\pi} E v \\
(\bar{\pi}-\underline{\pi}) v(1-\mu)-(1-\mu)(\bar{\pi}-\underline{\pi}) v \frac{(\bar{\pi}-\underline{\pi}) v+c}{c} & \geq \beta V(\mu ; \underline{\pi})-\frac{\beta}{1-\beta} \bar{\pi} E v \\
\frac{(1-\mu)((\bar{\pi}-\underline{\pi}) v)^{2}}{c} & \leq \frac{\beta}{1-\beta} \bar{\pi} E v-\beta V(\mu ; \underline{\pi}), \forall \mu \in\left[\mu^{*}(v), 1\right] .
\end{aligned}
$$

We will show later that this inequality is satisfied.

2. Now, suppose that $\mu^{*}(v) \geq \mu_{h}$. For $\mu^{*}(v)<\mu$, the proof is the same as before. So we only need to consider the case with $\mu_{h} \leq \mu<\mu^{*}(v)$. It is clear that no offer below the market-odds line attracts the high-quality bank. Furthermore, as before we can show that an offer of $\left(x_{h}^{\prime}, t_{h}^{\prime}\right)$ that is above the market-odds line attracts the low-quality bank as well, and hence the accompanying contract $\left(1, t_{l}^{\prime}\right)$ must be at least as good as $\left(x_{h}^{\prime}, t_{h}^{\prime}\right)$. That is,

$$
\begin{aligned}
t_{l}^{\prime}+\beta V(0 ; \underline{\pi}) & \geq t_{h}^{\prime}+\left(1-x_{h}^{\prime}\right)(\underline{\pi} v-c)+\beta V(1 ; \underline{\pi}) \\
t_{l}^{\prime} & \geq t_{h}^{\prime}-x_{h}^{\prime}(\underline{\pi} v-c)+\underline{\pi} v .
\end{aligned}
$$

So as before, we can show that this contract cannot make positive profits.

To conclude the proof, we show that the following inequality is satisfied:

$$
\frac{(1-\mu)((\bar{\pi}-\underline{\pi}) v)^{2}}{c} \leq \frac{\beta(1-\lambda)}{1-\beta(1-\lambda)} \bar{\pi} E v-\beta(1-\lambda) V(\mu ; \underline{\pi}), \forall v, \forall \mu \in\left[\mu^{*}(v), 1\right]
$$

Note that the right side of the above inequality is independent of $v$, so we have to check the above inequality at highest possible value of $v$. Now there are two possibilities. Either $\mu>\mu^{*}(\bar{v})$, in which case we only have to show that the inequality holds for $v=\bar{v}$, or 
$\mu \leq \mu^{*}(v)$, in which case we have to show the inequality for $v=v^{*}(\mu)$. Suppose that $v=\bar{v}$ and that $\mu>\mu^{*}(v)$. Note that since $v^{*}(\mu)>\bar{v}$, the equilibrium calls for selling at the pooling price all of the time. Hence, the above inequality becomes

$$
\frac{(1-\mu)((\bar{\pi}-\underline{\pi}) \bar{v})^{2}}{c} \leq \frac{\beta(1-\lambda)}{1-\beta(1-\lambda)}(1-\mu)(\bar{\pi}-\underline{\pi}) E v .
$$

Given our assumption above, this inequality is satisfied. Now, let us turn our attention to the second case where $\mu \leq \mu^{*}(v)$. In this case, the inequality becomes

$$
\frac{c}{1-\mu} \leq \frac{\beta(1-\lambda)}{1-\beta(1-\lambda)} \bar{\pi} E v-\beta(1-\lambda) V(\mu ; \underline{\pi}), \forall v, \forall \mu \in\left[\mu^{*}(v), 1\right],
$$

where the left side is evaluated at $v=v^{*}(\mu)$. We can rearrange the terms and write the above inequality as

$$
\frac{c}{1-\mu}+\beta(1-\lambda) V(\mu ; \underline{\pi}) \leq \frac{\beta(1-\lambda)}{1-\beta(1-\lambda)} \bar{\pi} E v .
$$

Since the left side is an increasing function of $\mu$, we need to show this inequality to be satisfied only at $\mu=\mu^{*}(\bar{v})$. Note that for $\mu=\mu^{*}(\bar{v})$, the equilibrium involves selling by all types all of the time; hence, $V(\mu ; \underline{\pi})=\frac{(\mu \bar{\pi}+(1-\mu) \underline{\pi}) E v}{1-\beta(1-\lambda)}$ and we can write

$$
\frac{c}{1-\mu^{*}(\bar{v})}=\frac{\left(1-\mu^{*}(\bar{v})\right)((\bar{\pi}-\underline{\pi}) \bar{v})^{2}}{c} .
$$

Hence, the above inequality can be written as

$$
\frac{\left(1-\mu^{*}(\bar{v})\right)((\bar{\pi}-\underline{\pi}) \bar{v})^{2}}{c} \leq \frac{\beta(1-\lambda)}{1-\beta(1-\lambda)}\left(1-\mu^{*}(\bar{v})\right)(\bar{\pi}-\underline{\pi}) E v .
$$

which is satisfied by our assumption above.

Q.E.D. 